\title{
Repensando o percurso metodológico de pesquisas etnográficas em tempos de pandemia: Uma breve revisão de literatura
}

\author{
Cátia Candido da Silva ${ }^{1}$ e Fabrícia Teixeira Borges ${ }^{2}$ \\ ${ }^{1}$ Secretaria de Estado de Educação do Distrito Federal / Universidade de Brasília, Brasil | \\ catiacandido77@yahoo.com.br | https://orcid.org/0000-0002-6236-7073 \\ 2Universidade de Brasília, Brasil | fabricia.borges@gmail.com | \\ https://orcid.org/0000-0002-9341-2738
}

\begin{abstract}
Resumo: Este artigo trata-se de uma revisão de literatura que teve como objetivo identificar como pesquisadores etnográficos solucionaram o impasse da necessidade de mudanças de percursos metodológicos impostas pelo distanciamento físico em decorrência da pandemia da Covid-19. Buscou-se identificar quais soluções foram propostas pelos estudiosos para a viabilização da continuidade das pesquisas. A Covid-19 é de uma doença nova com um alto poder de infecciosidade e difusão. Seu alastramento em dimensões mundiais levou a Organização Mundial de Saúde (OMS) a declarar pandemia e recomendar o distanciamento social como uma das medidas para a mitigação da sua disseminação. Tal acontecimento alterou o cotidiano da população acarretando na reorganização compulsória das configurações relacionais. Essas mudanças também impactaram a produção acadêmica, principalmente quanto à realização de pesquisas com abordagem etnográfica, pois a imersão no campo para a produção dos dados tornou-se inviabilizada. Nesse contexto, a pesquisa que temos em andamento foi afetada, uma vez que nosso objeto de estudo sustenta-se em elementos da constituição das subjetividades de crianças e adolescentes candomblecistas, o que havia nos levado a optar pela etnografia. Diante da necessária redefinição das rotas metodológicas, consideramos relevante identificar como outros pesquisadores solucionaram tal impasse. Para tanto, realizamos uma revisão de literatura com buscas no Portal de Periódicos Capes e no Scielo Brasil, utilizando os descritores etnografia e pandemia juntos. Analisamos 04 pesquisas empíricas que sofreram alterações na proposta metodológica em decorrência da pandemia da Covid-19. Como resultado, observou-se que os pesquisadores asseguraram a continuidade das pesquisas por meio da hibridização metodológica e da plataformização. Entre os instrumentos de pesquisa mais utilizados estão as redes sociais virtuais (Facebook e WhatsApp) e as plataformas de videoconferências (Jitsi Meet), além de formulários do Google Forms. Consideramos que os achados dessa revisão podem servir de inspiração para outros pesquisadores que necessitem alterar o método de suas pesquisas.
\end{abstract}

Palavras-chave: Metodologia; Etnografia; Pandemia; Covid-19.

Rethinking the Methodological Path of Ethnographic Research in Times of a Pandemic: A Brief Literature Review

Abstract: This article is a literature review that aimed to identify how ethnographic researchers solved the impasse of the need for changes in methodological paths imposed by physical distance due to the Covid-19 pandemic. We sought to identify which solutions were proposed by scholars to enable the continuity of research. Covid-19 is a new disease with a high infectivity and diffusion power. Its spread across the world led the World Health Organization (WHO) to declare a pandemic and recommend social distance as one of the measures to mitigate its spread. Such an event changed the daily life of the population, resulting in the compulsory reorganization of relational configurations. These changes have also impacted academic production, especially regarding the conduct of research with an ethnographic approach, as immersion in the field for the production of data has become unviable. In this context, the research that we have in progress was affected, since our object of study is based on elements of the constitution of the subjectivities of Candomblecist children and adolescents, which had led us to choose ethnography. In view of the necessary redefinition of the methodological routes, we consider it relevant to identify how other researchers solved this impasse. To this end, we conducted a literature review with searches on the Capes Periodicals Portal and Scielo Brasil, using the descriptors ethnography and pandemic together. We analyzed 04 empirical studies that underwent changes in the methodological proposal due to the Covid-19 pandemic. As a result, it was observed that the researchers ensured the continuity of the research through the methodological hybridization and the platform. Among the most used research tools are virtual social networks (Facebook and WhatsApp) and videoconferencing platforms (Jitsi Meet), in addition to Google Forms. We believe that the findings of this review may serve as inspiration for other researchers who need to change the method of their research.

Keywords: Methodology; Ethnography; Pandemic; Covid-19. 


\section{Introdução}

Este artigo trata-se de uma revisão de literatura que teve como objetivo identificar como pesquisadores etnográficos solucionaram o impasse da necessidade de mudanças de percursos metodológicos impostas pelo distanciamento físico em decorrência da pandemia da Covid-19. Buscou-se identificar quais soluções foram propostas pelos estudiosos para a viabilização da continuidade das pesquisas.

A Covid-19 é uma doença causada pelo coronavírus, denominado SARS-CoV-2, que apresenta um espectro clínico variando de infecções assintomáticas a quadros graves (Brasil, 2021). Trata-de de uma doença recente com um alto poder de infecciosidade e transmissibilidade que surgiu em dezembro de 2019 na cidade de Wuhan na China, sendo em seguida disseminada por todo o mundo. Seu alastramento em dimensões mundiais levou a Organização Mundial de Saúde (OMS) a declarar pandemia em março de 2020 e recomendar o distanciamento social como uma das medidas para a mitigação da sua disseminação. Tal acontecimento alterou o cotidiano da população acarretando na reorganização compulsória das configurações relacionais. Essas mudanças, evidentemente, também impactaram a produção acadêmica, principalmente quanto à realização de pesquisas com abordagem etnográfica, pois a imersão no campo para a produção dos dados tornou-se inviabilizada. Diante da inusitada situação de impossibilidade de continuação da produção de dados da forma inicialmente planejada, algumas questões surgiram: Como realizar etnografia em tempos de distanciamento físico? Como conhecer a cultura e compreender as interações entre as pessoas sem a possibilidade de interagir diretamente com elas?

\subsection{Sobre o Método Etnográfico}

O método etnográfico é um instrumento de pesquisa consagrado e amplamente utilizado em estudos realizados nos campos da antropologia, da educação, da sociologia, da comunicação, da psicologia e das inúmeras áreas de estudos centradas nos seres humanos e nas suas interações. Trata-se de um método que teve sua origem nos estudos antropológicos e que, paulatinamente, passou a ser utilizado de modo muito profícuo no desenvolvimento de estudos das mais diversas áreas que abordam questões relacionadas a subjetividades, alteridades, sistemas de valores, relações de gênero, religião, geracionalidade, entre outros (Souza, 2014).

Conforme Sato e Souza (2001), a etnografia trata-se de um método de pesquisa social por excelência, o qual considera que "qualquer descrição do comportamento humano requer a compreensão dos significados locais para descrevê-lo" (p. 32). Deste modo, essa abordagem metodológica demanda, no processo de pesquisa, a compreensão da cultura que está sendo estudada, não havendo a possibilidade de se dar dar a partir de procedimentos estandartizados. A este respeito, Fonseca (1999) arrazoa que, uma vez que cada povo ou grupo específico de sociedades complexas produz seus próprios valores culturais, de crença e dinâmicas sociais, ele não deve ser analisado pela ótica de outras culturas, com base em outras características e complexos axiológicos para que não se incorra no risco de haver interpretações enviesadas.

Nesse sentido, buscando compreender uma cultura específica e como os comportamentos, significados e processos sociais são construídos em cada grupo humano, os etnógrafos embrenham-se no cotidiano dos grupos pesquisados expondo-se longamente aos seus costumes e modos de vida. De acordo com Lima, Dupas, Oliveira e Kakehashi (1996) "o pesquisador deve realizar a maior parte do trabalho de campo pessoalmente, pois a experiência direta com a situação em estudo permite um contato íntimo e pessoal com a realidade estudada" (p. 25). Desta feita, entre os principais instrumentos de produção de dados utilizados na etnografia estão a observação participante, as entrevistas, as rodas de conversa e os grupos focais. 
Levando em consideração as características da abordagem etnográfica e a ampla gama de possibilidades de estudos que ela proporciona, havíamos optado pela etnografia para a construção dos dados da pesquisa que temos em andamento a qual objetiva analisar elementos da constituição das subjetividades de crianças e adolescentes candomblecistas. Para esse contexto, a etnografia configurava-se como uma excelente escolha metodológica, uma vez que os espaços religiosos das tradições de origem afrobrasileiras não são vistos apenas em sua dimensão religiosa, mas configuram-se, antes de tudo, como lugares de resistência da cultura e da história africanas, hospedando os saberes tradicionais. Desta feita, apenas a vivência cotidiana nos terreiros viabiliza o acesso ao conjunto de saberes fundamentais, possibilitando a compreensão da construção/ reconstrução dos saberes afro-referenciados e das múltiplas aprendizagens que participam da formação dos sujeitos.

Assim, com o objetivo de compreender a cosmovisão e as relações existentes nos terreiros de candomblé, a abordagem etnográfica foi escolhida como estratégia para a construção dos dados da nossa pesquisa. Desta feita, iniciamos o percurso metodológico por meio da observação participante em rituais abertos à comunidade em geral e, paulatinamente, passamos a vivenciar o cotidiano do terreiro não apenas nos momentos dos ritos, mas em vivências prolongadas. Nessas ocasiões, foi possível manter contato direto com os membros da comunidade e criar vínculos com aqueles que seriam os participantes da pesquisa. Durante as imersões nos terreiros, participamos colaborativamente dos afazeres na cozinha, da limpeza do quintal, da feitura das comidas de santo... Entretanto, em março de 2020, eclodiu a pandemia da Covid-19 e surgiu a eminente necessidade do afastamento das pessoas objetivando a mitigação do contágio, uma vez que trata-se de uma doença nova, altamente contagiosa e para a qual ainda não há tratamento específico comprovado. Por obviedade, nos afastamos das atividades do terreiro (que também foram suspensas), o que inviabilizou nosso trabalho e nos levou a buscar uma solução para a redefinição das rotas metodológicas.

\section{Uma Breve Revisão de Literatura}

\subsection{Metodologia do Estudo de Revisão}

$\mathrm{Na}$ tentativa de responder às questões levantadas e compreender como outros pesquisadores solucionaram o impasse da impossibilidade de realização da pesquisa etnográfica tradicional devido à pandemia do novo Coronavírus, empreendemos uma breve revisão de literatura para verificarmos quais reflexões foram realizadas e quais as soluções propostas para a adequação às novas configurações de modo a dar continuidade às pesquisas que inicialmente seriam realizadas por meio do método etnográfico. $\mathrm{Na}$ revisão sistemática empreendida buscamos seguir as seguintes etapas propostas pelo Instituto Cochrane (Gomes \& Carmina, 2014): (a) formulação da pergunta;(b) localização e seleção dos estudos em bases de dados indexadas; (c) avaliação crítica dos estudos; (d) coleta de dados; (e) análise e apresentação dos dados e (f) interpretação de dados. Para relatar o percurso da revisão, utilizamos alguns dos critérios recomendados pela diretriz Principais Itens para Relatar Revisões Sistemáticas e Meta-análises (PRISMA), conforme Galvão, Pansani e Harrad (2015).

Iniciamos a revisão de literatura realizando uma busca avançada no Portal de Periódicos Capes com a utilização dos descritores etnografia e pandemia juntos. Fizemos a opção por "artigos" em qualquer idioma para tipo de documentos, tendo obtido 38 artigos. Refinamos a pesquisa levando em consideração o período do início da pandemia e restringimos a periodização para artigos publicados entre os anos de 2019 e 2021 . Nesta busca, obtivemos 24 artigos.

Realizamos a leitura sistemática dos resumos dos 24 artigos e excluímos aqueles que não atendiam aos critérios de inclusão: a) se tratarem de pesquisas empíricas com seres humanos e b) terem sofrido alterações metodológicas em decorrência da pandemia da Covid-19. 
Assim sendo, foram descartados 8 artigos que se tratavam de editoriais, 3 que eram análises documentais, 1 revisão de literatura, 1 análise de obra literária e 4 ensaios filosóficos. Além disso, 2 estudos foram excluídos porque não reportavam alterações na metodologia e 2 não abordavam a pandemia da Covid-19. Deste modo, após a leitura dos resumos e o descarte daqueles estudos que não convergiam com nosso interesse, obtivemos apenas 3 artigos para a leitura na íntegra.

Como o resultado da busca por artigos acadêmicos no Portal Capes foi escasso, optamos por empreender uma pesquisa na plataforma Scientific Electronic Library Online - SciELO Brasil por tratar-se de uma biblioteca eletrônica que abrange uma coleção selecionada de periódicos científicos brasileiros e que nem sempre tem seus artigos reportados pelo Portal Capes. Utilizamos os mesmos termos, a mesma periodicidade e os mesmo critérios de inclusão e exclusão. Foram encontrados 4 artigos os quais tiveram seus resumos lidos. Destes, 2 estudos haviam sido iniciados em decorrência da pandemia, ou seja, não havia alterações metodológicas e 1 se tratava de um ensaio filosófico. Assim, apenas 1 dos artigos reportados por esta base teve sua leitura realizada na íntegra.

\subsection{Metodologia de Análise das Informações Extraídas das Fontes Selecionadas}

Para analisar os artigos, utilizamos a Análise de Conteúdo praticada por Bardin (2011) buscando seguir as etapas por ela propostas: 1) pré-análise; 2) exploração do material; 3) tratamento dos resultados e interpretação. Empreendemos a leitura sistemática dos textos completos, realizando as anotações pertinentes à nossa pesquisa. A leitura realizada enfocou a identificação dos objetivos, dos métodos e dos resultados encontrados nas pesquisas. A partir de então, em consonância com os objetivos da presente revisão, identificamos como categorias de análise emergentes: 1) Abordagem metodológica adotada; 2) Métodos iniciais de produção de dados; 3) Métodos de produção de dados durante a pandemia.

\subsection{Resultados e Análise}

Após as buscas realizadas nas duas bases de dados, considerando os artigos reportados pelo Portal de Periódicos Capes e pela plataforma Scielo Brasil, obtivemos, 4 artigos para serem analisados na íntegra, os quais foram organizados na Tabela 1.

Tabela 1. Artigos reportados pelas bases de dados.

\begin{tabular}{|c|c|c|c|c|}
\hline $\begin{array}{l}\text { Base } \\
\text { de } \\
\text { Dados }\end{array}$ & Descritores & Autores / Ano & Título & País \\
\hline \multirow[t]{3}{*}{$\begin{array}{l}\text { Portal } \\
\text { Capes }\end{array}$} & $\begin{array}{l}\text { etnografia } \\
\text { and } \\
\text { pandemia }\end{array}$ & $\begin{array}{l}\text { Sales, Cuevas- } \\
\text { Cerveró e Gómez- } \\
\text { Hernández (2020) }\end{array}$ & $\begin{array}{l}\text { Perspectives on the } \\
\text { information and digital } \\
\text { competence of Social } \\
\text { Sciences students and } \\
\text { faculty before and during } \\
\text { lockdown due to Covid-19 }\end{array}$ & Espanha \\
\hline & & $\begin{array}{c}\text { Bottino, Scheliga e } \\
\text { Menezes (2020) }\end{array}$ & $\begin{array}{l}\text { Experimentos etnográficos } \\
\text { em redes e varandas: a } \\
\text { religião em tempos de } \\
\text { pandemia }\end{array}$ & Brasil \\
\hline & & $\begin{array}{l}\text { Campos } \\
(2020)\end{array}$ & $\begin{array}{c}\text { Pesquisa etnográfica em } \\
\text { contextos digitais e de } \\
\text { isolamento social: desafios } \\
\text { metodológicos }\end{array}$ & Brasil \\
\hline $\begin{array}{l}\text { Scielo } \\
\text { Brasil }\end{array}$ & $\begin{array}{l}\text { etnografia } \\
\text { and } \\
\text { pandemia }\end{array}$ & $\begin{array}{c}\text { Sawaia, } \\
\text { Albuquerque e } \\
\text { Busarello (2020) }\end{array}$ & $\begin{array}{l}\text { O paradoxo do isolamento na } \\
\text { pandemia segundo o povo } \\
\text { indígena Sateré-Mawé/AM }\end{array}$ & Brasil \\
\hline
\end{tabular}


A análise dos artigos apontou que $100 \%$ dos estudos encontrados utilizaram a abordagem metodológica qualitativa com a utilização inicial de diferentes métodos de produção de dados. Observou-se que, inicialmente, havia a prevalência da proposição do emprego de observações participantes e entrevistas, conforme é possível observar na figura 1.

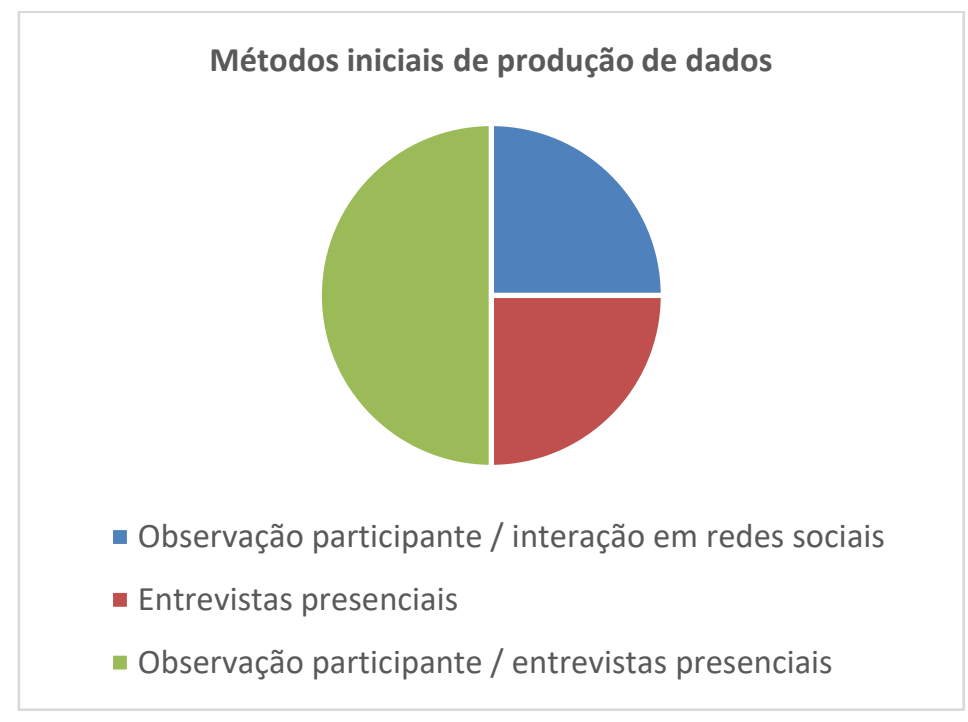

Fig. 1. Métodos iniciais de produção de dados.

Nota-se que $75 \%$ dos estudos analisados inicialmente propunham a utilização de observações participantes e/ou entrevistas presenciais, o que é característico de estudos etnográficos. Os outros $25 \%$ mesclavam a observação participante e a interação em redes sociais, ou seja, já faziam uso de uma metodologia híbrida.

Após a necessidade de alteração do percurso metodológico, $100 \%$ dos pesquisadores migraram para algum tipo de plataforma digital para dar continuidade à produção de dados de suas pesquisas, resultando em um processo de hibridização, conforme pode ser observado na figura 2 .

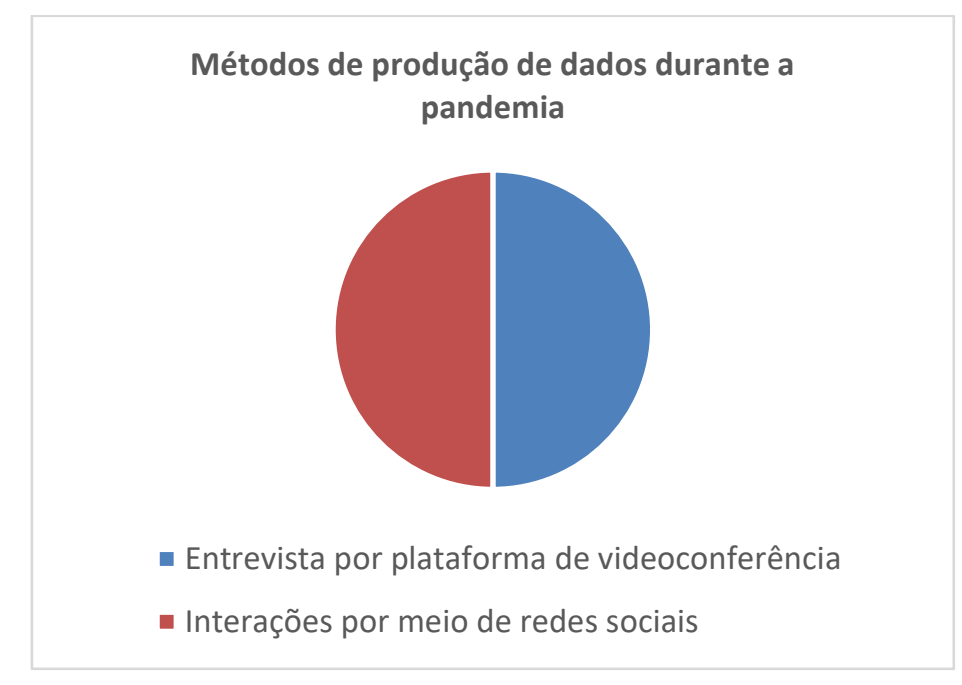

Fig. 2. Métodos de produção de dados durante a pandemia 
É possível constatar que os pesquisadores asseguraram a continuação das suas pesquisas por meio da hibridização metodológica. $50 \%$ dos estudiosos migraram para as plataformas digitais para a realização de entrevistas por videoconferência e a outra metade optou por dar continuidade à produção de dados por meio da utilização de redes sociais, a saber, Facebook e WhatsApp.

A seguir, relatamos brevemente os achados de cada um dos artigos de modo a explicitar especialmente as mudanças de percurso metodológico empreendidas. Os estudos foram categorizados a partir dos métodos de produção de dados adotados em decorrência da pandemia. $\mathrm{Na}$ análise, buscamos estabelecer um diálogo entre esses achados empíricos e as reflexões teóricas realizadas.

\subsubsection{Entrevistas por plataformas de videoconferência}

O estudo desenvolvido por Sales, Cuevas-Cerveró e Gómez-Hernández (2020) teve como o objetivo identificar como o corpo docente da área das ciências sociais percebia as competências informacionais e digitais dos estudantes, e se consideravam que eles próprios e seus alunos as dominam, a fim de identificar os problemas que condicionam. A metodologia utilizada foi híbrida em decorrência da pandemia do Coronavírus. Assim, o estudo foi realizado em duas etapas: a primeira sendo um grupo de discussão presencial entre os participantes (antes da pandemia) e a segunda uma entrevista realizada virtualmente com cada um dos professores (após o início do isolamento social). Os pesquisadores não especificaram qual plataforma de videoconferência foi utilizada. Embora tenha havido mudança metodológica devido à Covid-19, os autores não a apontaram como um problema que pudesse inviabilizar a produção dos dados. Aliás, segundo os autores, no decorrer da pesquisa, o evento crítico da pandemia foi incluído nos objetivos do estudo "porque forçou uma conversão acelerada para o modo virtual de ensino e foi capaz de revelar os níveis de domínio de competência dos alunos e professores" (Sales, Cuevas-Cerveró e Gómez-Hernández, 2020, p. 5). Os resultados da pesquisa demonstraram que os professores participantes do estudo possuem uma visão crítica acerca da competência informacional e digital dos estudantes. Eles apontam uma falta de capacidade de avaliação e de uso crítico e comunicação da informação do corpo discente, embora haja o domínio das aplicações tecnológicas.

Campos (2020) apresenta em seu artigo algumas impressões dos passos metodológicos utilizados na construção de uma pesquisa etnográfica diante da impossibilidade de realização de procedimentos de forma presencial devido às limitações de circulação de pessoas na pandemia de Covid-19. A autora relata que originalmente sua pesquisa propunha uma abordagem etnográfica viabilizada por meio de observação participante e de entrevistas com estagiárias em Jornalismo com o objetivo de compreender os entrelaçamentos entre suas trajetórias de vida, o mundo do trabalho e o ser jornalista. Diante da necessidade de adaptação dos procedimentos planejados inicialmente, Campos (2020) relata a migração metodológica para uma etnografia virtual, a qual já tem sido utilizada e discutida em pesquisas de diversas áreas (Amaral, Natal \& Viana, 2008; Polivanov, 2013), mas que, diante da pandemia da Covid-19, ganhou grande impulso. Dessa forma, para a construção dos dados, a pesquisadora recrutou as participantes por meio da rede social Facebook e utilizou o formulário do Google Forms e a plataforma de videoconferência Jitsi Meet para a realização das entrevistas. Refletindo sobre as potencialidades da pesquisa etnográfica plataformizada na produção de dados, Campos (2020) destaca a praticidade e a economia financeira e de tempo decorrentes do não deslocamento para o campo de pesquisa, a possibilidade de a pesquisa ser vista como menos invasiva, uma vez que o pesquisador não está presente no ambiente do participante, além, obviamente, da proteção à saúde de todos os envolvidos. Como desvantagens/ limitadores da plataformização da produção de dados, a autora sinaliza a menor autonomia do pesquisador em contatar os participantes (considerando que o algoritmo das plataformas faz conexões de pessoas de um determinado perfil), a inclinação ao esquecimento dos participantes para o compromisso virtual, as dificuldades de conexão à internet e de posse de equipamentos eletrônicos que oportunizem a atividade e a falta de contextualização. 


\subsubsection{Interações por meio de redes sociais}

No artigo "Experimentos etnográficos em redes e varandas: a religião em tempos de pandemia" as pesquisadoras Bottino, Scheliga e Menezes (2020) relatam uma pesquisa em curso com foco em experimentos etnográficos relacionados à religião em tempos de Covid-19. Nele são compartilhadas reflexões preliminares sobre arranjos e conciliações que produzem a religião vivida. As autoras explicam que já havia em curso uma pesquisa no campo de estudos da religião e áreas conexas, mas que o advento da pandemia as levou a realizar algumas notas etnográficas a respeito das interpelações que a Covid-19 tem provocado em redes e grupos dos quais as pesquisadoras participam (Facebook e WhatsApp). Assim, além da alteração metodológica, houve o surgimento do interesse por uma nova temática que dialogava com o que já era estudado. Desse modo, os dados da pesquisa foram produzidos por meio da etnografia híbrida, uma vez que ocorreu através da participação das pesquisadoras em redes sociais e aplicativos de mensagens (recursos que já eram utilizados anteriormente em pesquisas etnográficas realizadas pelas pesquisadoras) e da observação participante das interações que ocorriam entre as pessoas nas varandas em um condomínio onde uma das pesquisadoras reside. Conforme as autoras, em todos os ambientes observados foi possível constatar a multiplicação de práticas e a intensificação de celebrações religiosas durante a pandemia. Ademais, os experimentos por elas realizados demonstram que existe a possibilidade de continuidade das pesquisas antropológicas mesmo em situações de isolamento social, sendo necessárias algumas adaptações metodológicas.

O estudo de Sawaia, Albuquerque e Busarello (2020) relata parte de uma pesquisa que vem sendo desenvolvida desde $2019 \mathrm{com}$ comunidades tradicionais de quatro estados brasileiros: Rondônia, Amazonas, Santa Catarina e Piauí sob uma abordagem etnográfica. Com o advento da pandemia da Covid-19, os pesquisadores procuraram analisar e aprofundar interpretações acerca da relação entre desigualdade e pandemia em dimensão psicossocial, com foco no isolamento enquanto questão emblemática para indígenas Sateré-Mawé, do Amazonas. Nesse sentido, respeitando as recomendações da OMS, o percurso metodológico da pesquisa em curso foi alterado e a produção de dados específicos referentes à quarentena foi obtida por meio do uso de tecnologias de comunicação como redes sociais, mensagens pelo WhatsApp e telefonemas com lideranças indígenas e demais interlocutores de diversas etnias. Conforme os autores, além das narrativas obtidas por meios virtuais, as inferências interpretativas realizadas tiveram como base a análise de documentos públicos e na etnografia continuada que tem sido realizada com essas comunidades nos últimos oito anos. Sawaia, Albuquerque e Busarello (2020) argumentam que o isolamento social, apontado como único método preventivo de alta eficácia reconhecido pela OMS para a amenização da pandemia da Covid-19, tem sido, no caso dos povos indígenas, a estratégia ancestral utilizada para fugir de doenças de "gente branca", fazendo parte de sua sabedoria mítica. Desse modo, o modelo de isolamento territorial adotado pelos povos Sateré-Mawé e por, pelo menos 25 etnias em 13 territórios federais, começou a ser desenvolvido no início de abril de 2020 com adesões paulatinas e voluntárias. Tal ação preventiva foi viabilizada por meio de Redes de comunicação organizadas pelas próprias etnias. Porém, conforme os autores, paradoxalmente, o isolamento contra doenças traz "o risco de se agregar comorbidades relacionais à própria peste, por conta da política brasileira que permite invasões a terras nativas por interesses de poder, impedindo a proteção de lugares de refúgio, como faziam ancestrais do continente sul-americano" (Sawaia, Albuquerque e Busarello, 2020, p. 7). Os autores concluem seu estudo afirmando que, além de toda a gravidade para a saúde da população inerente à doença, a pandemia tem ativado medos históricos nas sociedades nativas, situados a partir de violências vivenciadas por elas no passado e no presente. Em contrapartida, o evento também evidenciou a valorização de conhecimentos ancestrais que, historicamente foram e têm sido capazes de salvar vidas. 


\section{Conclusões}

Embora o número de estudos encontrado nessa breve revisão de literatura tenha sido exíguo, sobretudo em virtude da recenticidade da temática, consideramos que as soluções apresentadas pelos pesquisadores para solucionarem o impasse da impossibilidade de realização da produção de dados por meio da etnografia podem contribuir para que outros estudiosos também deem andamento em suas pesquisas. A revisão evidenciou o despontamento dos processos de hibridização metodológica e de plataformização das pesquisas com o crescente uso de etnografia virtual e da netnografia, indicando a necessidade de uma maior apropriação dos pesquisadores sobre essas temáticas que vêm ganhando cada vez mais espaço na produção acadêmica. Além disso, os resultados da revisão proporcionaram uma reflexão sobre o uso de novas tecnologias em pesquisa qualitativa e a necessidade de flexibilização metodológica por parte do pesquisador de modo a considerar as mudanças nos contextos e nas condições sociais.

Identificamos como limitação desta revisão a escassez de resultados numéricos obtidos, uma vez que pretendíamos analisar apenas pesquisas já concluídas e descritas em artigos publicados e indexados nas bases de dados. No entanto, embora nosso objetivo não fosse avaliar e acompanhar alterações metodológicas de pesquisas em curso, consideramos mister mencionar que muitas discussões e estudos estão sendo realizadas desde o início da pandemia no sentido de ampliar o debate sobre as adaptações metodológicas e dar suporte a pesquisadores para a manutenção de suas produções científicas. Notadamente podemos citar o relatório Rapid Evidence Review publicado pela National Centre for Research Methods que trata-se de um estudo colaborativo o qual buscou mapear como os métodos de pesquisa social estão sendo adaptados com sucesso ou projetados para uso nas condições pandêmicas da Covid-19. Para fins de aprofundamento, disponibilizamos nas referências o link para tal relatório.

\section{Referências}

Amaral, A. R., Natal, G., \&Viana, L. (2008). Netnografia como aporte metodológico da pesquisa em comunicação digital. Sessões do Imaginário, 20, 34-40.

Bardin L. (2011).Análise de conteúdo. Lisboa: Edições 70.

Barros, O. C., \& Serpa Jr., O. D. (2017). Ouvir vozes: Um estudo netnográfico de ambientes virtuais para ajuda mútua. Physis Revista de Saúde Coletiva, 27 (4), 867-888. http://dx.doi.org/10.1590/S0103-73312017000400002

Brasil (2021). Coronavírus (Covid-19): Sobre a doença. Retirado de https://coronavirus.saude.gov.br/sobre-a-doenca

Bottino, C. M. M., Scheliga, E., \& Menezes, R. C. (2020). Experimentos etnográficos em redes e varandas: A religião em tempos de pandemia. Cadernos de Campo (online), 29 (suplemento), 289-301.

Campos, E. T. M. (2020). Pesquisa etnográfica em contextos digitais e de isolamento social: Desafios metodológicos. Intercom - Sociedade Brasileira de Estudos Interdisciplinares da Comunicação. 43ํㅡㄹ Congresso Brasileiro de Ciências da Comunicação, Brasil.

Corrêa M. V., \& Rozados H. B. F. (2017). A netnografia como método de pesquisa em ciência da informação. Encontros Bibli: Revista eletrônica de Biblioteconomia e Ciência da Informação, 22 (49), 1-18. https://doi.org/10.5007/1518-2924.2017v22n49p1

Fonseca, C. (1999). Quando cada caso não é um caso: Pesquisa etnográfica e educação. Revista Brasileira de Educação,10, 58-78.

Galvão, T., Pansani, T., \& Harrad, D. (2015). Principais itens para relatar revisões sistemáticas e meta-análises: A recomendação PRISMA. Epidemiologia e Serviços de Saúde, 24(2), 335342. doi:10.5123/S1679-49742015000200017

Gomes, I. S., \& Carmina, I. O. (2014). Guia para estudos de revisão sistemática: Uma opção metodológica para as Ciências do Movimento Humano. Movimento, 20 (01), 395-411. 
Lima, C. M. G., Dupas, G., Oliveira, I., \& Kakehashi, S. (1996). Pesquisa etnográfica: Iniciando sua compreensão. Revista Latino-americana de enfermagem, 4 (1), 21-30.

Nind, M., Coverdale, A. \& Meckin, R. (2020). Changing Social Research Practices in the Context of Covid-19: Rapid Evidence Review. Economic and Social Research Council.

Polivanov, B. B. (2013). Etnografia virtual, netnografia ou apenas etnografia? Implicações dos conceitos. Esferas - Revista Interprogramas de Pós-graduação em Comunicação do Centro Oeste, 2, 61-71.

Sales, D., Cuevas-Cerveró, A., \& Gómez-Hernández, J. A. (2020). Perspectives on the information and digital competence of Social Sciences students and faculty before and during lockdown due to Covid-19. Profesional de la información, 29(4), 1-21. https://doi.org/10.3145/epi.

Sato, L., \& Souza, M. P. R. (2001). Contribuindo para desvelar a complexidade do cotidiano através da pesquisa etnográfica em Psicologia. Psicologia USP, 12 (2), 29-47.

Souza, M. R. (2014). Uma questão de método: Origens, limites e possibilidades da etnografia para a psicologia social. Psicologia USP, 25(3), 307-316. https://doi.org/10.1590/0103 656420130038. 